\title{
The Interface between Psychiatry and Ophthalmology
}

\author{
Evgenii Sadykov ${ }^{1,3, *}$, Jan Studnička², Ladislav Hosák¹,3, Mikela-Rafaella Siligardou ${ }^{4}$, Hajer Elfurjani ${ }^{4}$, Jessica \\ Leung Hoikam4, Surentheran Kugananthan ${ }^{4}$, Anastasios Petrovas ${ }^{4}$, Talal Amjad ${ }^{4}$
}

\section{ABSTRACT}

Objective: The aim of this article is to review the interface between psychiatry and ophthalmology at several levels, such as the influence of psychopharmacology on eye disorders, the occurrence of psychiatric symptoms in eye diseases, and the neuroophthalmological examination methods supporting the validity of psychiatric diagnoses.

Materials and Methods: We searched the PubMed computer database for the key words "Psychiatry" and "Ophthalmology" on the 28th of August, 2018 to obtain relevant articles which were consequently summarized.

Results: The results showed that most patients with ocular disease simultaneously have one or more psychiatric symptoms. We also found a prevalence of eye-related side effects in patients who use psychiatric drugs. At the same time, we observed that some ophthalmology methods of diagnostics can be used as diagnostic tools in psychiatry.

Conclusions: Most studies showed a significant relation between psychiatry and ophthalmology, such as eye symptoms and diseases following long-term use of psychotropics as well as psychiatric symptoms and syndromes in patients with eye disorders. Our review may be beneficial to psychiatrists, ophthalmologists, and, last but not least, the patients themselves.

\section{KEYWORDS}

depression; eye disease; psychopharmacology; dry eye syndrome; schizophrenia; blepharitis; bipolar disorder; pharmacotherapy

\section{AUTHOR AFFILIATIONS}

${ }^{1}$ Department of Psychiatry, Charles University, Faculty of Medicine in Hradec Králové, Czech Republic

2 Department of Ophthalmology, Charles University, Faculty of Medicine in Hradec Králové, and University Hospital Hradec Králové, Czech Republic

${ }^{3}$ Department of Psychiatry, University Hospital Hradec Králové, Czech Republic

${ }^{4}$ Charles University, Faculty of Medicine in Hradec Králové, Czech Republic

* Corresponding author: Department of Psychiatry, Charles University, Faculty of Medicine in Hradec Králové, Šimkova 870, 50038 Hradec Králové, Czech Republic; e-mail: evgenii.sadykov@fnhk.cz

Received: 3 March 2019

Accepted: 13 May 2019

Published online: 26 July 2019

Acta Medica (Hradec Králové) 2019; 62(2): 45-51

https://doi.org/10.14712/18059694.2019.104

(c) 2019 The Authors. This is an open-access article distributed under the terms of the Creative Commons Attribution License (http://creativecommons.org/licenses/by/4.0), which permits unrestricted use, distribution, and reproduction in any medium, provided the original author and source are credited. 


\section{INTRODUCTION}

Mental disorders can be a manifestation of an organic brain disease - tumor, cyst, inflammatory process, traumatic encephalopathy, cerebral atherosclerosis, etc. The diagnostic of the cerebral process is important for a psychiatrist. Neuroophthalmological methods, with their results frequently reflecting brain pathology, include studies of acuity and field of vision, movements of the eyeballs, pupillary reactions, and the eye fundus. The results of neuroophthalmological diagnostics could potentially influence the psychiatric diagnosis and treatment strategy.

The aim of this article is to review the interface between psychiatry and ophthalmology at several levels, such as the influence of psychopharmacology on eye disorders, the occurrence of psychiatric symptoms in eye diseases, and the neuroophthalmological examination methods supporting the validity of a psychiatric diagnosis. This may be helpful for psychiatrists as well as ophthalmologists, from the clinical and scientific point of view.

\section{MATERIALS AND METHODS}

We searched the PubMed computer database for the key words "Psychiatry" and "Ophthalmology" on the 28th of August 2018 to get relevant articles. Afterwards, we summarized their content. We preferred large studies to individual case reports and recent findings to a historical knowledge. Only articles in English were considered for this review.

\section{RESULTS}

\section{DEPRESSION AND ANXIETY IN OPHTHALMOLOGY}

Chiang et al. (2013) conducted a retrospective follow-up observation to estimate the risk of depression and anxiety in patients with blepharitis using nationwide insurance data from 1997 to 2010 in Taiwan to identify annually patients with newly diagnosed blepharitis $(\mathrm{N}=9764)$ and those without the disease $(\mathrm{N}=39056)$. A chi-squared test was used to examine the distributions of baseline demographic characteristics and comorbidities between blepharitis and non-blepharitis patient groups. The results show that the incidence rate of anxiety and depression were (1.67-fold (95\% CI $=1.46-1.70)$ and 1.29 -fold ( $95 \%$ $\mathrm{CI}=1.28-1.58)$ respectively) higher in the blepharitis cohort than in the non-blepharitis cohort. It was also higher in women than in men and increased with age in both cohorts (1).

Zhou et al. (2013) studied the prevalence of depression and anxiety symptoms in Chinese patients with glaucoma. The study included 506 patients with glaucoma. In the study the authors used the Hospital Anxiety and Depression Scale (HADS) and the Chinese-version of the Glaucoma Quality of Life-15 questionnaire (CHI-GQL-15). Multiple linear regression analyses were used to identifying the predictors of depression and anxiety. The results showed that in Chinese glaucoma patients the prevalence of depression and anxiety was $16.4 \%$ and $22.92 \%$, respectively. The study also showed that independent predictors of anxiety were female gender $(P=0.001)$, younger age $(\mathrm{P}<0.001)$, moderate and heavy economic burden $(\mathrm{P}=0.009 ; \mathrm{P}<0.001$ respectively), and the summary score of the CHI-GQL-15 (P<0.001). The CHI-GQL-15 summary score $(\mathrm{P}<0.001)$ and the duration of glaucoma $(\mathrm{P}=0.019)$ were significant independent predictors of glaucoma. The authors concluded that, among Chinese patients with glaucoma, the prevalence of depression and anxiety was relatively high (2).

Chen et al. (2015) evaluated the risk of primary open-angle glaucoma (POAG) and primary angle-closure glaucoma (PACG) in depression patients with long-term use of selective serotonin reuptake inhibitors. The authors used data from the National Health Insurance Research Database in Taiwan and identified 26,186 patients with newly diagnosed depression and without preexisting glaucoma. The authors divided the patients into two cohorts. The first cohort included 13,093 patients with one year of serotonin reuptake inhibitors use. The second was a comparison cohort which included 13,093 patients who had never used serotonin reuptake inhibitors. All cohorts included patients $>20$ years. The authors used the Kaplan-Meier method for evaluating the incidence of open-angle and angle-closed glaucoma between serotonin reuptake inhibitors and the comparison cohorts. For calculating the differences between the curves, the authors used a long-rank test. It was revealed that the incidence of open-angle and angle-closure glaucoma between the serotonin reuptake inhibitors and comparison cohorts had non-significant differences (long-rank test $\mathrm{P}=0.52$ for open-angle glaucoma, $\mathrm{P}=0.32$ for angle-closure glaucoma). Also, the authors concluded that the incidence of open-angle glaucoma in the serotonin reuptake inhibitors cohort was non-significantly higher than that in the comparison cohort (1.51 vs 1.39 per 1000 person-years), with an adjusted hazard ratio of $1.07(95 \% \mathrm{CI}=0.82-1.40)$. The incidence of angle-closure glaucoma in the serotonin reuptake inhibitors was non-significantly lower than that in the comparison cohorts (0.95 vs 1.11 per 1000 person-years), with an adjusted hazard ratio of 0.85 (95\% CI $=0.62-1.18$ ). The authors concluded that the risk of primary open-angle and primary closed-angle glaucoma in the Chinese ethnic population in Taiwan does not depend on the long-term use of serotonin reuptake inhibitors (3).

The purpose of the Vaart et al. (2015) study from the University of North Carolina was to analyze the association between dry eye disease and depression and anxiety. The cases of anxiety (ICD-9 code $300,300.00,300.01$, 300.02), depression (ICD-9 code 311, 296.2x, 296.3x), dry eye disease (ICD-9 code 375.15) and rheumatoid arthritis (ICD-9 code 714.0) were defined according to ICD-9 codes. The authors were interested in the associations between the diagnosis of dry eye disease and each of depression and anxiety. Because of it they separately estimated odds ratio between dry eye and each of the other disease. For estimating the associational ratio the authors used separate logistic regression models. A total of 460,611 patients were screened; 7,207 patients with dry eye were included, as were 20,004 patients with anxiety and 30,100 patients with depression. All patients were over the age of 18 . 
Separate odds ratios were calculated for dry eye disease, for anxiety, and for depression. The results show the estimated odds ratio between dry eye disease and anxiety was 2.8 (95\% CI 2.6-3.0), while the ratio between dry eye disease and depression was 2.9 (95\% CI 2.7-3.1). The study identified statistically significant associations between dry eye and the psychiatric diseases of depression and anxiety in a large population study (4).

In another study, to detect at which level of visual impairment psychological symptoms might appear, Palagyi et al. (2016) from Australia evaluated the prevalence and predicators of depressive symptoms in 329 older patients (aged $\geq 65$ years) who were on a waiting list for cataract surgery. For measuring symptoms of depression, the authors used the 5-item Geriatric Depression Scale (GDS-5). Visual disability was assessed by the Catquest-9SF, visual acuity was measured binocularly and was corrected by the high contrast Early Treatment Diabetic Retinopathy Study (ETDRS) and binocular contrast sensitivity was measured by the Mars Letter Contrast Sensitivity Test. All measurements were done at least one month prior to surgery. Following a complete assessment, 94 patients (28.9\%) were shown to have depressive symptoms. The depressive symptoms were significantly associated with visual disability $(P=0.020)$, higher comorbidity score $(P=0.020)$, and reduced quality of life $(P=0.003)$. The study demonstrated a high prevalence of depressive symptoms in older persons with cataract. The authors recommended creating an efficient referring system in order to reduce the surgery waiting time and to improve care coordination. This should include the screening and management of depressive signs during the surgery waiting time, which will play a great role in minimizing the negative psychological impacts of a cataract-related loss of vision in this group of patients (5).

Wan et al. (2016) from China assessed the association of depression and anxiety with dry eye disease by conducting a meta-analysis and systematic review that reported the incidence and prevalence of anxiety and depression in patients with dry eye disease versus healthy controls. The authors analyzed 22 studies consisting of 2,980,026 patients. The meta-analyses showed that dry eye disease was associated with an increased prevalence of anxiety $(\mathrm{OR}=2.80,95 \%, \mathrm{P}<0.00001)$ and depression $(\mathrm{OR}=2.92$, $\mathrm{P}<0.00001)$. In patients with dry eye disease, the anxiety score $(P=0.007)$ and depression score $(P<0.00001)$ were significantly higher than in controls (6).

\section{BIPOLAR DISORDER IN OPHTHALMOLOGY}

Mehraban et al. (2016) from Iran measured peripapillary retinal nerve fiber layer thickness (RNFLT) in 30 patients with bipolar disorder and 30 age-matched healthy controls. The authors measured the mean RNFLT of the 4 quadrants by using Optical coherence tomography (OCT). The results showed that mean RNFLT was significantly less in the case group than in the controls, $99 \pm 8$ and $106 \pm 8 \mu$, respectively $(P=0.001)$. The significant difference in RNFLT was in the superior $(P=0.040)$, inferior $(\mathrm{P}<0.001)$, and nasal $(\mathrm{P}=0.005)$ quadrants in both the case and control groups. The temporal quadrant was not reduced significantly compared to the control group $(P=0.907)$. The authors discovered that the duration of bipolar disorder had a variable significant relation with RNFLT. The authors concluded that measuring the reduction of the peripapilary retinal nerve fibre layer thickness in patients with bipolar disorder can be of beneficial value when studying neurodegenerative changes (7).

O'Bryan et al. (2015) evaluated the disturbances of visual motion perception in 61 patients with type I bipolar disorder (40 women, 21 men, mean age $=40$ ) and 67 comparison subjects ( 35 women, 32 men, mean age $=39$ ). The authors used psychophysical tests of contrast sensitivity, form discrimination, and dot motion discrimination for investigating visual perception in bipolar disorder. The results showed a deficit in dot motion trajectory discrimination in both euthymic and ill individuals with bipolar disorder $(P=0.007)$ and a nearly significant difference in global deficit in moving grating contrast sensitivity $(\mathrm{P}=0.054)$. Patients with bipolar disorder were impaired in psychomotor processing but the result was not related to visual processing performance (8).

\section{SCHIZOPHRENIA IN OPHTHALMOLOGY}

The review by Gracitelli et al. (2015) showed that most studies on schizophrenia have focused on disturbances in higher-order brain functions associated with the frontal and pre-frontal cortex. However, recently there have been reported abnormalities in low-level sensory processes, such as the visual system. At early stages of the disease, patients report to have visual disturbances in perception. It is the variations in the dopamine and glutamate systems that have been highlighted in the pathophysiology of the disease. Also, the loss of retinal nerve fiber layer thickness has been well established in several neurologic diseases which also involve dopamine and glutamate dysregulation (9). Thirty schizophrenia patients and 30 agematched controls were studied with spectral-domain optical coherence tomography and it was found that these measurements were reduced in schizophrenia patients. Cortical visual pathways have been described as magnocellular pathways, parvocellular pathways, and koniocellular pathways. Several studies have reported that visual impairment in schizophrenia patients could result from dysfunction in the magnocellular pathway. Tilt After Effects (TAE) in chronic schizophrenia patients were studied using neuroleptic drugs and compared with Parkinson's disease patients (9). It was shown that, if presenting peripheral fine gratings, schizophrenia patients produced results similar to those suffering from Parkinson's. It has been reported that schizophrenia patients make more color discrimination errors than control subjects. Studies concerning eye movements in schizophrenia patients have found reduced pursuit gain, low initial acceleration, and abnormal gain-corrective saccade interactions (9). Schizophrenia is associated with consistent deficits in visual processing in the early stage of the disease. It is therefore possible for ophthalmological assessment to be carried out as a further aid in the diagnosis of schizophrenia (9).

Ascaso et al. (2010) measured the retinal nerve fiber layer (RNFL) thickness by optical coherence tomography in 
10 patients (males -8 , females -2 , mean age $39+/-13$ years) with schizophrenia. The patients were from the Department of Psychiatry at the Hospital Clinico Universitario in Zaragoza, Spain. All patients had a corrected visual acuity of $20 / 20$ (with a refractive error of $+/-2$ spheric diopters) and intraocular pressure < than $18 \mathrm{mmHg}$. The group of patients was compared with ten age-matched controls. Peripapillary RNFL thickness, optic nerve head (ONH) measurements, macular thickness and volume were measured by optical coherence tomography (OCT) in both the patients and control group. The results showed a statistically significant reduction of the overall RNFL thickness in schizophrenic patients compared with controls $(\mathrm{P}=0.047)$ and reduced peripapillary RNFL thickness in nasal quadrant in schizophrenic patients compared to controls $(P=0.048)$. No statistically significant differences were observed between schizophrenia patients and the control group with regard to ONH measurements and macular thickness and volume. The authors showed a statistically significant reduction of peripapillary RNFL thickness in schizophrenia patients evaluated by OCT (10).

\section{SLEEP DISORDER IN OPHTHALMOLOGY}

Ayaki et al. (2016) studied sleep and mood disorders in 715 Japanese patients with irritating ocular surface disease, 301 patients with dry eye disease, and 202 age matched healthy controls. Patients filled out the Pittsburgh Sleep Quality Index (PSQI) and the Hospital Anxiety and Depression Scale (HADS) questionnaires. Photophobia as the major symptom of dry eye disease was measured with two representative questionnaires (National Eye Institute Visual Function Questionnaire-25 and Morning/Eveningness questionnaires). The analyse showed that scores, respectively, for PSQI and HADS were $5.0 \pm 3.3$ and $8.9 \pm 5.3$ for allergic conjunctivitis $(n=78), 5.5 \pm 3.1$ and $9.5 \pm 6.6$ for chronic conjunctivitis $(\mathrm{n}=124), 6.4 \pm 3.2$ and $11.1 \pm 5.7$ for severe dry eye disease $(n=146)$, and $5.5 \pm 3.3$ and $9.8 \pm 4.0$ for mild dry eye disease $(n=155)$. There was a significant difference among these diagnostic groups for PSQI $(\mathrm{P}<0.05)$. The severity of dry eye disease significantly correlated with the PSQI and HADS $(P<0.05)$. The authors of the study demonstrated that sleep quality in patients with dry eye disease was significantly worse than in patients with other irritating ocular diseases, thus indicating a correlation with the severity of dry eye disease (11).

Liguori et al. (2016) performed an electrophysiological study of the visual system in patients with severe obstructive sleep apnea (OSA) in 6 women and 21 men (mean age $44+/-10$ ) without medical comorbidities compared to a healthy control group (10 women, 17 men, mean age 41 +/-11). The authors evaluated the integrity of the visual system by means of electroretinogram (ERG) and visual evoked potential (VEP). Day time sleepiness was assessed by using the Epworth Sleepiness Scale (ESS). The results showed a significant latency delay coupled with a significant amplitude reduction of P100 wave of VEP at all spatial frequencies in both eyes in patients with OSA compared to the control group $(\mathrm{P}<0.001)$. No significant differences between patients with OSA and healthy controls were detected concerning ERG components. The authors also failed to find correlations between VEP and ERG components or polygraphic parameters and ESS scores in patients with OSA. The study showed that patients with OSA presented VEP alterations by a lower amplitude and longer latency of the P100 component than healthy controls. These altered electrophysiological findings may be the result of optic nerve dysfunction, which was provoked by airway obstruction, hypoxia, hypercarbia and acidosis, conditions frequently observed in patients with obstructive sleep apnoea (12).

\section{PEDOPSYCHIATRY IN OPHTHALMOLOGY}

Cumurcu et al. (2011) measured social phobia and other psychiatric problems in 42 children 8-13 years with strabismus and 47 healthy controls from Turkey. For evaluating social phobias, the authors used the Screen for Child Anxiety Related Emotional Disorders (SCARED). For each child, the researchers used the Children's Depression Inventory (CDI) and Schedule for Affective Disorders and Schizophrenia for School-Aged Children-Present and Lifetime Version (Kiddie-SADS-PL). The results showed that social phobia was diagnosed in $8(19.04 \%)$ of the $42 \mathrm{pa}-$ tients with strabismus and in $1(2.12 \%)$ of the healthy controls. Significantly higher were the CDI $(P=0.001)$ and SCARED (total score $(P=0.004)$, social phobia $(P=0.0001)$ and separation anxiety $(\mathrm{P}=0.05)$ scores of strabismus patient than the control group. The authors concluded that there was a relationship between anxiety, depression, social phobia and children with strabismus, as evidenced by their data (13).

Karaman et al. (2016) in Turkey measured the effect of eye trauma on the mental health and quality of life in 20 patients aged 5 to 18 and their parents. For evaluating the quality of life for both parents and children, the authors used the Pediatric Scale of Quality of Life (PedsQL), and for evaluating mental health the Affect Disorders and Schizophrenia Interview Chart for school children, now and lifelong (AFSIC-NL), was used, along with the Child Post-Traumatic Stress Reaction Index (CPTSD-RI). 95\% of patients were exposed to penetrating trauma and $1 \%$ to blunt. Nine patients were diagnosed with a mental disorder such as major depression (3.15\%), posttraumatic stress disorder (3.15\%), and generalized anxiety disorder (3.15\%) according to AFSIC-NL. The authors found a statistically significant relationship between lens damage or initial visual acuity and the PedsQL-parent emotional functional scale. Also, the parents of patients had a mild posttraumatic stress disorder regarding the CPTSD-RI. The authors concluded that eye trauma can lead to the development of psychopathology in children (14).

Ikeda et al. (2013) evaluated the incidence of ophthalmologic disorders in 154 (79\% male and 21\% female) children with autism from the USA, all examined between 1998 and 2006. For analyzing the data, the authors used a SPSS statistical program. The subgroups of patients in the autistic spectrum were: $44.16 \%$ mild to moderate autism, $20.13 \%$ pervasive developmental disorder, $13.64 \%$ autism spectrum disorder, $11.04 \%$ severe autism, $7.14 \%$ moderate to severe autism, 3.25\% Asperger's, 0.64\% Rett syndrome. The findings showed that children with autism had as 
follows: no ophthalmic problem $(\mathrm{N}=61, \mathrm{p}=0.002)$, strabismus $(\mathrm{N}=32, \mathrm{p}=0.001)$, amblyopia $(\mathrm{N}=16, \mathrm{p}=0.127)$, refractive error $(\mathrm{N}=44, \mathrm{p}=0.044)$. No relation was found with autism for children who had a history of prematurity gestation (<37 weeks). The findings suggest that ophthalmologic disorders were common in children with diagnoses in the autism spectrum (15).

Black et al. (2013) made a retrospective chart review of ocular abnormality in 44 patients (3:1 males to females, ages ranging 2 to 20 years) diagnosed with autism spectrum disorders (ASD) between January 2007 and October 2011 in the Greater Baltimore Medical Center (GBMC), USA. The results of the review showed that $52 \%$ (23) of patients with ASD at GBMC had some sort of ocular abnormality: strabismus $41 \%$ (18), significant refractive error $27 \%$ (12), anisometropia $7 \%$ (3), and amblyopia $11 \%(5)$. The authors found that the prevalence of amblyopia, anisometropia, and strabismus were higher among patients with ASD seen at the GBMC pediatric-ophthalmology practice than in the general population (16).

\section{GERONTOPSYCHIATRY IN OPHTHALMOLOGY}

$\mathrm{Su}$ et al. (2016) investigated the association between primary open-angle and primary angle-closure glaucoma with the risk of dementia in 6509 patients with glaucoma (3304 with open-angle and 3025 with angle-closure glaucoma) and a comparison cohort of 26,036 individuals without glaucoma and dementia in Taiwan. For evaluating the risk of dementia, univariate and multivariate Cox proportional hazard models were used. The research showed a higher risk of dementia in patients with glaucoma than in individuals without glaucoma (HR $=1.13,95 \%$, $\mathrm{CI}=1.01-1.27)$. The patients with open-angle glaucoma had a 1.21-fold increased risk of dementia compared with the individuals without glaucoma $(\mathrm{HR}=1.21,95 \%, \mathrm{CI}=1.02$ 1.43). On the other hand, the patients with angle-closure glaucoma were not significantly associated with an increased risk of dementia compared with the healthy individuals ( $\mathrm{HR}=1.09,95 \%, \mathrm{CI}=0.95-1.26)$. Patients aged $\geq 65$ years with open-angle glaucoma were significantly associated with an increased risk of dementia compared to the healthy individuals ( $\mathrm{HR}=1.28,95 \%, \mathrm{CI}=1.07-1.54)$. The authors concluded that patients with open-angle glaucoma were significantly associated with an increased risk of dementia compared with healthy individuals but not those with angle-closure glaucoma (17).

\section{PSYCHOPHARMACOLOGY IN OPHTHALMOLOGY}

A medical case report by Sears et al. (2015) suggested that long-term use of chlorpromazine can induce conjunctival secondary bilateral melanosis with unilateral limbal tumor (18). Chlorpromazine can also cause pigmentary or granular retinosis, benign natured pigmentary segregation on the lens or cornea as well as corneal edema (Sonmez and Aykan 2014) (19). Another case report by Karadaget al. (2015) indicated that the use of the antipsychotic drug aripiprazole can cause an acute transient bilateral myopia. However, the effect was reversed when patients replaced the drug (20). Similarly, Costagliola et al. (2008) suggested the use of SSRIs would increase intraocular pressure, induce optic nerve head changes, and predispose patients to angle closure, but this is dependent on each patient's individual tolerability of SSRIs (21). Additionally, SSRIs also affects the pupil diameter, aqueous humor dynamics, and optic nerve blood flow. In the review of Sonmez and Aykan (2014), a list of typical psychiatric drugs and their ocular side effects were described. These consist of thioridazine, butyrophenones, TCAs, benzodiazepines, mood stabilizers, lamotrigine, topiramate, and lithium. The side effects include retinitis pigmentosa, visual impairment, accommodation disorder, various eye movements disorder, nystagmus, and even oculogyric crisis (19).

The study of Borovik et al. (2009) refers to a case of a 55-year old female with a history of schizophrenia, depression, hypothyroidism, gastro-esophageal reflux and back pain. She was being treated with high dose of clozapine for 16 years and her cumulative dose was $4.67 \mathrm{~kg}$, lithium carbonate $500 \mathrm{mg}$ daily, thyroxine $100 \mu \mathrm{g}$ daily and omeprazole $20 \mathrm{mg}$ daily. She presented with bilateral pigmentary changes in her cornea and retina and stellate cataract. Clozapine is recommended in patients who experience pigmentary changes from chlorpromazine use, changes which are expected to resolve. The reason phenothiazine-related ocular changes happen is not known, but it is suspected to be related to photosensitization of tissues in which the drug has accumulated after sun exposure or that they alter the dopaminergic regulation of melatonin, which is suspected to increase the susceptibility of photoreceptors to damage by light. In this patient, it may be possible that these changes were produced by the high doses of clozapine, which also acts on dopamine receptors, since none of her other medications are known to have any such side effects (22).

The study of Emoto et al. (2011) was conducted to see whether psychotropic cessation in patients with drug-induced blepharospasm improves motor symptoms. Part or all of the patients' psychotropic medication was withdrawn and their motor symptoms using the Jankovic rating scale were assessed. Twelve patients were sampled where psychotropics were administered before the onset of blepharospasm in all patients. The mean duration of treatment ranged between 3 and 120 months. At initial presentation, the Jankovic rating scale was 3 in eleven patients and 2 in one patient. After the cessation of medication, blepharospasm improved in all cases within 2 months. Therefore, it was concluded that blepharospasm was directly caused by the psychotropic agents (23).

Stein et al. (2015) measured the risk of open-angle glaucoma and bupropion use in 638,481 patients $\geq 35$ years with no pre-existing open-angle glaucoma. For assessing the impact of bupropion and other antidepressant medications on the risk of open-angle glaucoma, the authors used the multivariable Cox regression modelling. The results showed that open-angle glaucoma developed in 15,292 patients (2.4\%). Each additional month of bupropion use was associated with a $0.6 \%$ reduced risk of open-angle glaucoma $(\mathrm{P}=0.007)$ after adjustment for use of other antidepressant medication. Patients with 24-48 months of bupropion use had a $21 \%$ reduced hazard $(P=0.0099)$ of open-angle glaucoma compared to non-users. The study 
showed that the development of open-angle glaucoma was not significant associated with using selective serotonin reuptake inhibitors $(\mathrm{P}=0.39)$ and tricyclic antidepressants $(\mathrm{P}=0.95)$. The authors concluded that bupropion use may be beneficial in reducing the risk of open-angle glaucoma (24).

In a cross-sectional observational study by Acan et al. (2016), the authors aimed to determine the influence of selective serotonin reuptake inhibitors (SSRIs) on ocular surface and tear film stability. 36 patients (Group 1) and 36 healthy volunteers (Group 2) were enrolled (25). Group 1 was composed of patients in clinical remission of depression and/or anxiety to exclude the possible effect of the psychiatric disease on ocular disease when patients were using SSRIs. Other exclusions included subjects with other psychiatric or systemic disease, the usage of medication or contact lenses, pregnancy, lactation or ocular disease history for the past 12 months. Both Group 1 and 2 underwent detailed psychiatric and ophthalmological examination. The results showed no significant differences between the groups in terms of age and sex $(P>0.05)(25)$. The most commonly used SSRI among Group 1 was fluoxetine and the period of usage ranged between 2 and 120 months. It is not clearly seen whether the psychiatric disease or the medication used for its treatment were associated with the development of dry eye symptoms. Antidepressants could induce dry eye disease, as most of them inhibit amine (norepinephrine and serotonin) reuptake pumps, thus increasing functional amine-dependent synaptic transmission. In addition, serotonin receptors have been identified in the corneal and conjunctival epithelia, meaning that altered concentrations of serotonin by the use of SSRIs could affect the eyes (25).

Three cases of intraoperative floppy-iris syndrome (IFIS) were reported by Matsuo et al. (2016) during cataract surgery in patients using long-term antipsychotic drugs but without a history of selective al blockers (26). IFIS is a complication that may occur during cataract extraction in certain patients. This syndrome is characterized by a flaccid iris which billows in response to ordinary intraocular fluid currents, a propensity for this floppy iris to prolapse towards the area of cataract extraction during surgery, and progressive intraoperative pupil constriction despite standard procedures to prevent this (27). The first case refers to a 39-year old male with chronic angle-closure glaucoma who was being treated for schizophrenia with several classes of antipsychotic drugs, including haloperidol, chlorpromazine, aripiprazole, olanzapine, quetiapine, risperidone, and blonanserin for more than 7 years and topical $\beta$-blocker timolol for the glaucoma. In the second case, a 63-year old female was being treated for schizophrenia for more than 10 years with aripiprazole, quetiapine, and risperidone and the topical $\alpha \beta$-blocker levobunolol to treat glaucoma. In the last case, a 65-year old male had a history of haloperidol use for more than 10 years for an organic mental disorder and the topical $\beta$-blocker timolol for the treatment of glaucoma, as in case 1 (26). There are also 5 more cases reported in the literature with use of long-term chlorpromazine, risperidone, and quetiapine. All three cases presented with incomplete IFIS, no history of selective al-blocker for glaucoma, and antipsychotic drug use. Even though antipsychotic drug related IFIS is not common, it seems that any class of antipsychotic drugs can cause such problems as they all have antagonistic effects on acetylcholine, histamine, and $a$-adrenergic receptors as side effects. Surgeons should take heed of this (26).

\section{DISCUSSION AND CONCLUSION}

An ophthalmological examination is part of the complex somatic and paraclinical examination of patients suffering from mental disorders. From previous articles, we can recognize the close relationship between psychiatry and ophthalmology. Unfortunately, only a small number of physicians examine their patients for side effects of psychotropic and ophthalmology drugs or level of anxiety before surgery. The possible value of ophthalmological symptoms and findings for a diagnosis and treatment of mental diseases is determined by the close anatomical and functional connection of the eye and the brain, by the commonality of their vascular system, and a high perceptibility of the eye to the side effects of psychotropic drugs. In an outpatient setting, neuroopthalmological examinations can be easily performed because they are usually cheap, quick, non-invasive, painless and widely available.

Most of the reviewed studies showed a significant relation between psychiatry and ophthalmology, such as eye symptoms in mentally ill subjects, mental problems accompanying eye diseases, and significant adverse side effects of psychotropics on the eye. Psychiatrists should be able to recognize signs and symptoms of eye disorders in people with mental problems, and ophthalmologists should be skilled enough to recognize symptoms of psychiatric disorders in their patients. An early recognition of symptoms in these two fields of medicine can help to start an adequate complex therapy and increase the quality of life of psychiatric as well as ophthalmological patients.

\section{CONFLICT OF INTEREST STATEMENT}

The authors declare they have no conflicts of interest.

\section{ACKNOWLEDGEMENTS}

Sponsored by the Ministry of Health of the Czech Republic, Czech Health Research Counsil, research grant AZV MZ ČR 16-27243A.

\section{REFERENCES}

1. Chiang CC, Lin CL, Tsai YY, Peng CL, Liao YT, Sung FC. Patients with blepharitis are at elevated risk of anxiety and depression. PLoS One 2013 Dec 30; 8(12): e83335.

2. Zhou C, Qian S, WU P, Qiu C. Anxiety and depression in Chinese patients with glaucoma: Sociodemographic, clinical, and self-reported correlates. J Psychosom Res 2013 Jul; 75(1): 75-82.

3. Chen HY, Lin CL, Kao CH. Long-term use of selective serotonin reuptake inhibitors and risk of glaucoma in depression patients. Medicine (Baltimore) 2015 Nov; 94(45): e2041. 
4. Vaart R, Weaver MA, Lefebvre C, Davis RM. The association between dry eye disease and depression and anxiety in a large population-based study. Am J Ophthalmol 2015 Mar; 159(3): 470-4.

5. Palagyi A, Rogers K, Meuleners L, et al. Depressive symptoms in older adults awaiting cataract surgery. Clin Exp Ophthalmol 2016 Dec; 44(9): 789-96.

6. Wan $\mathrm{KH}$, Chen LJ, Young AL. Depression and anxiety in dry eye disease: a systematic review and meta-analysis. Eye (Lond) $2016 \mathrm{Dec}$; 30(12): 1558-67.

7. Mehraban A, Samimi SM, Entezari M, Seifi MH, Nazari M, Yaseri M. Peripapillary retinal nerve fiber layer thickness in bipolar disorder. Graefes Arch Clin Exp Ophthalmol 2016 Feb; 254(2): 365-71.

8. O'Bryan RA, Brenner CA, Hetrick WP, et al. Disturbances of visual motion perception in bipolar disorder. Bipolar Disord 2014 June 16(4): 354-65.

9. Gracitelli CPB, Abe RY, Diniz-Filho A, Vaz-de-Lima FB, Paranhos A Medeiros FA. Ophthalmology Issues in Schizophrenia. Curr Psychiatry Rep 2015 May; 17(5): 28.

10. Ascaso FJ, Cabezon L, Quintanilla MA, et al. Retinal nerve fiber layer thickness measured by optical coherence tomography in patients with schizophrenia: A short report. Eur J Psychiat 2010; 24(4): 227-35.

11. Ayaki M, Kawashima M, Negishi K, Kishimoto T, Mimura M, Tsubota K. Sleep and mood disorders in dry eye disease and allied irritating ocular diseases. Sci Rep 2016; 6: 22480.

12. Liguori CL, Palmieri MG, Pierantozzi M et al. Optic Nerve Dysfunction in Obstructive Sleep Apnea: An Electrophysiological Study Sleep 2016; 39(1): 19-23.

13. Cumurcu T, Cumurcu BE, Ozcan O, Demirel S, Duz C, Porgali E, et al. Social phobia and other psychiatric problems in children with strabismus. Can J Ophthalmol 2011 Jun; 46(3): 267-70.

14. Karaman S, Ozkan B, Gok M, Karakaya I, Kara O, Altintas O, et al. Effect of eye trauma on mental health and quality of life in children and adolescents. Int Ophthalmol 2016 Jul; 37(3): 539-44.
15. Ikeda J, Davitt BV, Ultmann M, et al. Brief Report: Incidence of Ophthalmologic Disorders in Children with Autism. J Autism Dev Disord 2013; 43: 1447-51.

16. Black K, McCarus C, Collins MLZ, et al. Ocular Manifestations of Autism in Ophthalmology. Strabismus 2013; 21(2): 98-102.

17. Su CW, Lin CC, Kao CH, Chen HY. Association between glaucoma and the risk of dementia. Medicine (Baltimore) 2016 Feb; 95(7): e2833.

18. Sears KS, Rennie IG, Mudhar HS. Unilateral conjunctival in situ squamous carcinoma with bilateral conjunctival chlorpromazine-induced secondary melanosis masquerading as in situ and invasive melanoma. Ocul Oncol Pathol 2015 Jun; 1(4): 231-6.

19. Sonmez I, Aykan U. Psychotropic drugs and ocular side effects. Turk J Ophthalmol 2014; 44: 144-50.

20. Karadag H, Acar M, Ozdel K. Aripiprazole induced acute transient bilateral myopia: a case report. Balkan Med J 2015 Apr; 32(2): 230-2.

21. Costagliola C, Parmeggiani F, Semeraro F, Sebastiani A. Selective serotonin reuptake inhibitors: a review of its effects on intraocular pressure. CurrNeuropharmacol 2008 Dec; 6(4): 293-310.

22. Borovik AM, Bosch MM, Watson SL. Ocular pigmentation associated with clozapine. Med J Aust 2009 Feb 16; 190(4): 210-1.

23. Emoto Y, Emoto H, Oishi E, Hikita S, Wakakura M. Twelve cases of drug-induced blepharospasm improved within 2 months of psychotropic cessation. Drug Healthc Patient Saf 2011; 3: 9-14.

24. Stein JD, Talwar N, KangJH, Okereke OI, Wiggs JL, Pasquale LR. Bupropion use and risk of open-angle glaucoma among enrollees in a large U.S. managed care network. PLoS One 2015 Apr 13; 10(4): e0123682.

25. Acan D, Kurtgoz P. Influence of selective serotonin reuptake inhibitors on ocular surface. Clin Exp Optom 2017; 100(1): 83-6.

26. Matsuo M, Sano I, Ikeda Y, Fujihara E, Tanito M. Intraoperative floppy-iris syndromeassociatedwithuse of antipsychoticdrugs. Can J Ophthalmol 2016 Aug; 51(4): 294-6.

27. Chang D, Campbell J. Intraoperative floppy iris syndrome associated with tamsulosin. J Cataract Refract Surg 2005; 31(4): 664-73. 